254, L59.

Tanaka, K., Nitta, N., and Watanabe, T.: 1982b, in Hinotori Symp. on Solar Flares (ISAS: Tokyo, Japan), pp. 20-23.

Tanaka, K., Akita, K., Watanabe, T., and Nishi, K.: 1982c, in Hinotori Symp. on Solar Flares (ISAS: Tokyo, Japan), pp. 43-57.

Tanaka, K., Nitta, N., Akita, K., and Watanabe, T.: 1983, Solar Phys. 86(1/2), 91-100.

Tanaka, K., Watanabe, T., and Nitta, N.: 1984, Astrophys. J. 282, 793.

Tsuneta, S.: 1984, Astrophys. J. (in press).

Tsuneta, S., Takakura, T., Nitta, N., Ohki, K., Makishima, K., Murakami, T., Oda, M., and Ogawara, Y.: 1983, Solar Phys. 86(1/2), 313-321.

Tsuneta, S., Takakura, T., Nitta, N., Ohki, K., Tanaka, K., Makishima, K., Murakami, T., Oda, M., Ogawara, Y., and Kondo, I.: 1984a, Astrophys. J. 280, 887-891.

Tsuneta, S., Nitta, N., Ohki, K., Takakura, T., Tanaka, K., Makishima, K., Murakami, T., Oda, M., and Ogawara, Y.: 1984b, Astrophys. J. 284, 827-832.

Watanabe, T., Tanaka, K., Akita, K., and Nitta, N.: 1983, Solar Phys. 86(1/2), 107-113.

Yoshimori, M., Okudaira, K., Hirasima, Y., and Kondo, I.: 1983, 18th Internat. Cosmic Ray Conf. Vol. 4, pp. 85-88 (Tata Inst. Fundamental Res.: Bombay).

\title{
V. Sunspots
}

(v. Bumba)

Several proceedings of scientific meetings on sunspots appeared during the 1981-1984 period [The Physics of Sunsots, Cram and Thomas (eds.) 1981; see also reports of regional meetings, e.g., Third European Solar Meeting, Oxford 1981; Nordic Astronomy Meeting, O. Hauge (ed.), Oslo 1983; 11th Regional Consultation on Solar Physics, L. Dezsö and B. Kalman (eds.), Debrecen 1983]. New interest in sunspots was aroused through observations of EUV sunspot spectra from space and was also inspired by the growing number of observations of starspots and other stellar activities [IAU Symposium No. 102, solar and stellar Magnetic Fields: Origin and Coronal Effects, J.O. Stenflo (ed.) 1983; Colloquium IAU No. 71 Activity in Red Dwarf Stars, Catania 1982]. Other reasons for the increased interest in sunspots and their energetics were prompted by the correlation between sunspot occurrence and the variations of the solar constant (Hudson et al. 1982) and by the use of sunspot positions for determining solar differential rotation and its change with latitude, depth, and time (Howard et al. 1984, Godoli \& Mazzucconi 1982, Balthasar et al. 1984, Tuominen \& Kyröläinen 1982, Adam 1983, Koch 1984).

\section{A. EVOLUTION OF SUNSPOTS IN THE FRAME OF ACTIVE REGION'S MAGNETIC FIELD}

Current studies define with more precision than before the modes of sunspot development from individual nuclei, sunspot rotation, and the course of penumbral formation, strongly dependent on field topology, etc. (Bumba \& Suda 1983a, 1984a,b, Liggett \& Zirin 1983). The studies describe the complicated changes in umbral and penumbral forms due to the action of colliding magnetic entities of the same or opposite polarities (Bumba et al. 1982). The structure of the magnetic field and its evolution around sunspots visualized in photospheric and chromospheric fine structures has been studied during the normal development of sunspots (Gopasyuk \& Kartashova 1981), in cases of more complicated magnetic situations (Karlicky \& Suda 1983), during the appearance of " $\delta$ spots" (Patty 1981, Tang 1983), and during still more complex magnetic field topologies (Lin \& Wang 1982, Yang \& Chang 1983), as well as in association with flare occurrence (Ikhsanov 1982). The investigation of the behavior of sunspot magnetic flux during the time of sunspot disappearance brought a new enigma concerning the manner in which sunspot magnetic fields vanish. Some observers (Wallenhorst \& Howard 1982, Wallenhorst \& Topka 1982) do not see any evidence for spreading or diffusion of the field into the supergranular network, although others see small 
spots and pores as the result of secondary effects caused by the formation (Parfinenko 1983) or the decay (Garcia de la Rosa 1981, 1983) of larger active regions.

B. HIGH RESOLUTION AND SPECTRAI OBSERVATIONS OF THE PHOTOSPHERE, CHROMOSPHERE, AND TRANSITION REGION IN UMBRAE AND PENUMBRAE

The study of bright umbral dots continued. Adjabshirzadeh \& Koutchmy (1982) demonstrated that the dots do not show the foreshortening effect typically observed in granular structures near the solar limb. The same authors (Adjabshirzadeh \& Koutchmy 1983) constructed from their spectrophotometrical measurements a preliminary model of a two-component umbra. Parfinenko (1981a) measured the mean contrast of bright points; Soltan (1981) demonstrated a morphological relation between umbral dots and bright penumbral filaments. Lawrence (1983), using a statistical analysis, showed that umbral dots mapped by different observers have different distributions, probably due to the different resolution of the instruments used. Moore (1981a) proposed that umbral dots and penumbral grains are essentially the same phenomenon. Abdusamatov (1980) concluded that bright umbral points cover about 108 of the total umbral area and that their corrected mean brightness is smaller than that of the undisturbed photosphere. From new observations of umbral limb-darkening Albregtsen et al. (1984) found a significant decrease in the umbra-photosphere intensity ratio toward the limb. Wittmann (1983) derived corrected relative intensities of umbral dots, penumbral filaments, pores, and photospheric granulation as a function of wavelength. Van Ballegooijen (1984) constructed models of the temperature stratification in the deep layers of the umbra from a high-resolution infrared spot spectrum. Only a few papers appeared dealing with molecular spectra were studied (Glenar et al. 1983, wöhl et al. 1983, sinha 1983, Sinha \& Joshi 1983, Lee et al. 1982a,b, Yun \& Kim 1983). Abdusamatov (1984) demonstrated with the use of photometric studies the absence of the exterior as well as of the interior bright rings in studied sunspots. Two main types of sunspot 1ightbridges have been confirmed by Parfinenko (1981b), and links of Ha-flare emission above sunspots with these light-bridges have been shown (Bumba et al. 1981). With the help of the speckle image reconstruction procedures stachnik et al. (1983) demonstrated that the finest structures in sunspots appear to be embedded in penumbral filaments. The mean width and distribution of these filaments were estimated during the solar eclipse of June 1973 (Bonet et al. 1982). A good sumnary of properties of penumbral fine structures was published by Muller (1981). Rapid variations of contrast with time of penumbral bright features were found by Parfinenko (1982). Moore (1981b) concluded that some of the dark filaments located at the outex edge of a well observed penumbra may lie several hundred kilometers above the base of the quiet photosphere. This concept has been theoretically (Cram et al. 1981) and observationally (Lites 1984) developed but needs further observational confirmation, because many observed details of sunspot structure are not in good mutual agreement (Bumba \& suda 1983b).

Using the repeated intensity and velocity images of a large isolated sunspot in both a chromospheric and a photospheric line, Lites et al. (1982) showed a relationship between the propagating umbral disturbances and the running penumbral waves: the oscillations in the sunspot are very regular and the phase relationship between the velocity and intensity of the chromospheric oscillations is radically different from that of the quiet Sun. Ye $J$ in $(1981 a, b)$ found that spectral lines formed in the deep layers of a sunspot may be notably influenced by Alfvén waves, while this influence is weak on lines originating in its upper layers. The widths and intensities of $\mathrm{Ca}$ II $\mathrm{H}$ and $\mathrm{K}$ emission lines across a sunspot were estimated ( $Y u n$ et al. 1982, Yun \& Beebe 1982), and Gurman (1984) published observations of the Mg II $H$ line in sunspot umbrae. The dependence of umbral flashes on the magnetic field structure of sunspots and other parameters was established (Turova et al. 1983, Turova 1984, Teplitskaya \& Turova 1983). slow and fast variations in the brightness oscillations of a sunspot umbra in the 
center of the $H \alpha$ line were shown (Loskutnjkov 1984). From observations of brightness oscillation in a sunspot umbra in both the $\mathrm{H \alpha}$ and $\mathrm{Ca}$ II $\mathrm{K}$ lines, a model was constructed which explains the oscillations by resonant transmission of slow-mode magnetoacoustic waves semi-trapped in a chromospheric cavity above the umbra (Zhugzhda \& Makarov 1982, Zhugzhda et al. 1984). Many papers trying to explain the umbral oscillations and photospheric and chromospheric penumbral waves were published, e.g., Cally \& Adam 1983, Zhugzhda et al. 1983, Thomas \& scheuer 1982, and Cally 1983). Spectra of several strong lines obtained in sunspots (Beebe et al. 1982) allowed discussions of models of the chromosphere above a sunspot umbra or penumbra (Yun et al. 1984). The observations of intensities of $L \alpha$ and $L \beta$ above sunspots yielded estimates of the pressure in the upper chromosphere and transition region above the umbra, the values of wich are somewhat lower than the average quiet coronal pressure (Lites \& skumanich 1981). The UV observations from the SMM satellite gave the physical and dynamic characteristics of the transition region above sunspots: low intensities, mass flow, electron densities (Kingston et al. 1982), presence of both subsonic and supersonic flow, reduced electron temperature, and energy budget (Nicolas et al. 1982). Oscillations with periods of about $2 \mathrm{~min}$ of line-of-sight velocity and intensity of transition-region lines were observed (Gurman et al. 1982, Henze et al. 1984).

\section{OBSERVATIONS OF MAGNETIC FIELDS}

Huseynov (1983) studied the Zeeman splitting of Fe II and Fe I lines in several sunspots. Fluctuations of up to 1000 Gauss between the individual bright and dark penumbral regions were found by Stellmacher \& weihr (1981). Brants \& Zwaan (1982), measuring the magnetic field strengths in small umbrae and pores using the $T 1$ I line formed in umbrae, found values between 1900-2600 Gauss in their darkest parts. Using different temperature-sensitive lines Huseynov \& Russkih (1983) studied the distribution of field strength across the umbrae. The degree of polarization for $\pi$-components of two photospheric lines in sunspot umbrae was estimated by Vyalshin \& Kulagin (1981). The circular polarization of sunspots with high sensitivity $\left(\sim 3 \times 10^{-6}\right)$ in a wide spectral range (3700-4500 $\AA$ ) was measured by Kemp \& Henson (1983); in the same way Faraday rotation during measurements of the orientation of sunspot transverse magnetic field was investigated in detail (West \& Hagyard 1983). The influence of instrumental polarization on such measurements was discussed by Kawakami (1983). Coordinated ground-based and SMM satellite observations of sunspot magnetic fields were carried out by (Hagyard et al. 1983), and (Henze et al. 1982). Koval \& Stepanjan (1983), estimated that there are several modes of magentic field distribution with height above sunspots. Obashev et al. (1982) estimated the angle of inclination of the sunspot symmetry axis to the solar surface from obsevations of the Wilson depression. Giovanelli \& Jones (1982) studying the magnetic canopies above sunspots, demonstrated that these canopies extend from sunspots at a height of a few hundred kilometers above the outer edge of the sunspot penumbrae beyond the sunspots, rising slowly, almost horizontally, into the height of several hunared kilometers interconnecting various spots.

\section{OBSERVATIONS OF MOTIONS IN AND ABOVE SUNSPOTS}

Tönjes and wöhl (1982) confirmed that penumbral bright grains lasting 1.5 to 3 hours move toward sunspot umbrae with velocities of 80 to $330 \mathrm{~m} \mathrm{~s}^{-1}$. The motions in different depths of the umbral photosphere were studied by Adam (1983) and Copasyuk (1982). From space observations of spectral lines originating in the transition region above sunspots Athay et al. (1982) demonstrated the steady flow patterns in the reversed Evershed sense with a substantial vertical component. Nicolas (1981) detected systematic flows in the EUV spectrum that extend to about $10^{4} \mathrm{~km}$ outside the penumbra. Moore (1981c) presented his model of dynamic phenomena in sunspots, for which Thomas (1981) attempted to. find a theoretical explanation within the framework of magnetohydrodymamic theory. Spruit (1981a) reviewed the role of convective processes in the small-scale 
umbral and penumbral phenomena. Several papers were devoted to the study of spot motions in sunspot groups (e.g., Doval 1983). Two types of such motions were discussed by Dezsö \& Kovacs (1983). Quasiperiodic oscillations of relative distances of small spots and pores in a group were found by selenzneva (1981). Bumba \& Suda (1983c) pointed out the low efficiency of magnetic forces during the formation, motion, and collision of sunspots and the importance of hydrodynamic forces during these processes. The irregular rotation of several spots was studied by Kučera (1982) and Antalova (1983).

\section{E. RADIO ASTRONOMICAL OBSERVATIONS OF SUNSPOTS}

Measurements estimating the physical conditions above sunspots, especially the magnetic field intensity and structure with the aid of radio astronomical methods, have been made by Abramov-Maksimov \& Gelfreikh (1983), and Bogod et al. (1982) demonstrated that the magnetic field intensity above sunspots at the bottom of the corona either coincided with the photospheric value or was smaller by about 30\%. Akhmedov et al. (1982a) studied the s-component of the solar radio emission and showed that the height of its sources above umbrae did not generally exceed $2000 \mathrm{~km}$. They also estimated the magnetic field gradient which is not less than 0.25 Gauss $\mathrm{km}^{-1}$. Akhmedov et al. (1982b, 1983) compared radio observations with model calculations. Comparing the radio data with uv and soft $x$-ray data Shibasaki et al. (1983) identified the radio emission mechanisms and their height and magnetic field strength above sunspots. The absence of polarized emission above the sunspot umbra and the circularly polarized emission above the penumbra were detected at $6 \mathrm{~cm}$ by Lang \& Willson (1982). Additional radio emission above bipolar sunspot group was observed by Akhmedov \& Gelfreikh (1981).

\section{F. THEORETICAL MODELS OF SUNSPOTS}

Yoshimura (1983) presented a new concept of sunspot cooling based on the size and depth of magnetic field tubes. Albregtsen \& Maltby (1981) drew attention to the possibility of distinguishing between different sunspot theories by observing the umbra/photosphere intensity ratio as a function of spot size, and Elboth \& Engvold (1983) estimated the contrast of this ratio for a large sunspot with high spectral resolution. Avrett \& Kurucz (1983) used the $3860-3880 \AA$ band for a detailed study of the sunspot/photosphere intensity ratio and suggested that in sunspots it is formed at the temperature minimum with a brightness temperature of about $3350 \mathrm{~K}$. Schatten (1981) proposed as a sunspot cooling mechanism the gravitational draining in which the magnetic field of pores provides a conduit wherein cooled gases can return more easily to their heat source at the bottom of the convective zone. Staude (1981) derived horizontally averaged working models for both the umbra of a large stable spot and its mean undisturbed environment for reference. Fowler et al. (1983) studied the intensity distribution around sunspots for comparison with the isotherms predicted by convective blocking models of heat flow. The magnetic field played a dominant role in many newly proposed sunspot models. Solovev (1982) examined the force-free magnetic flux rope with the uniform twisting in which the cross-section radius of the magnetic surface varies along the symmetry axis of the system. Huseynov (1982) looked for sunspot models and conditions where equilibrium between the total pressure of a sunspot and the pressure of the surrounding photosphere is reached. Spruit (1981b) reviewed the current theories concerning the pressure equilibrium and thermal balance of sunspots, their hydromagnetic stability, energy transport mechanisms, and wave propagations. Solovev (1983) discussed the problem of static confinement in magnetic flux tubes of variable diameter.

A new magnetohydrostatic theory of sunspots, taking into account the magnetic field lines which return to the photosphere, was discussed by Osherovich (1982). Flaa et al. (1982) and Osherovich (1984) applied this model to a typical sunspot; Osherovich \& Flaa (1983) included the azimuthal component of the magnetic field into the model, which only slightly influenced the sunspot 
structure. Another extension of this model into a "double return flux model" was presented by Osherovich \& Lawrence (1983). In a series of papers Solovev (1981, 1982b, 1983) and Mordvinov (1983) discussed the physical behavior of a boundary layer between the sunspot magnetic flux tube and the photosphere under different assumptions. A theoretical analysis of torsional oscillations of sunspots in a bipolar group observed by Gopasyuk (1981) was presented by Solovev (1984). From a derived simple relation between the period of oscillations and the effective depth of the sunspots, a depth of a few thousand kilometers was estimated. Thomas et al. (1982) interpreted the observations of sunspot umbra 5-min oscillations splitting into several individual modes of different periods as the response of the sunspot to forcing by the 5 -min p-modes in the surrounding quiet atmsophere. Thomas (1984) interpreted the fundamental 3-min umbral oscillations as due to a photospheric resonance. His theory of umbral oscillations was supported by calculating the resonant modes in a much more detailed model of the umbral atmsophere (Thomas \& Schever 1982). Bel \& Leroy (1981) showed that the cooling of sunspots by ascending Alfvén waves is not possible. Krivodubskij (1983), studying the efficiency of diffexent mechanisms of sunspot magnetic field dissipation, found that in the deep layers of the sunspot umbra the Alfvén oscillations are prevalent, while in the upper layers ohmic dissipation seems to be more effective. singh et al. (1983), investigating the dynamical properties of convective overstability and Kelvin-Helmholtz instability in a vertical magnetic field with a downdraft, demonstrated that both agents produce upward/downward propagating Alfvén waves depending on the magnitude of the kinematic viscosity coefficient and the thermometric conductivity. The electric current density in a sunspot was calculated from the computed distribution of the magnetic field vector compared with its actual distribution in a real spot by Denisenko et al. (1982). Similar computations of the distribution of vertical electric current density were compared with UV emissions by deloach et al. (1984). The electrical conductivity, thermal conductivity, and viscosity of models both of the quiet photosphere and of the sunspot umbra were calculated by Kovitya \& Cram (1983).

\section{References}

Abdusamatov, K.I.: 1980, Soln. Dann. 11, 99.

Abdusamatov, K.I.: 1984, Soln. Dann. 6, 80 .

Abramov-Maksimov and Gelfretkh, G.B.: 1983, Pisma Astron. Zh. 9, 244.

Adam, M.G.: 1983, M.N.R.A.S. 203, 9-18.

Adjabshirzadeh, A. and Koutchmy, S.: 1982, Solar Phys. 75, 71-74.

Adjabshirzadeh, A. and Koutchmy, S.: 1983, Astron. Astrophys. 122, 1-8.

Akhmedov, Sh. B. and Gelfreikh, G.B.: 1981, Soln. Dann. 6, 107.

Akhmedov, Sh. B., Gelfretkh, G.B., Bogod, V.M., and Korzhavin, A.N.: 1982a, Solar Phys. 79, 41-58.

Akhmedov, Sh. B. et al.: 1982b, Soln. Dann. 10, 72 .

Akhmedov, Sh. B., Gelfreikh, G.B., Fürstenberg, F., Hilderbrant, J., and Krüger, A.: 1983, Solar Phys. 88, 103-108.

Albregtsen, F. and Maltby, R.: 1981, Solar Phys. 74, 147-151.

Albregtsen, F., Joras, P.B., and Maltby, P.: 1984, Solar Phys. 90, 17-30.

Antalova, A.: 1983, Bull: Astron. Inst. Czech. 33, 345.

Athay, R.G., Gurman, J.B., Henze, W., and Shine, R.A.: 1982, Astrophys. J. 261, 684 .

Avrett, E.H. and Kurucz, R.L.: 1983, Rep. Inst. Theoret. Astrophys., Blindern, Olso 59, 43-58.

Balthasar, H., Lust1g, G., and wöhl, H.: 1984, Solar Phys. 91, 55-59.

Beebe, H.A., Baggett, W.E., and Yun, H.S.: 1982, Solar Phys. 79, 31-39.

Bel, N. and Leroy, B.: 1981, Astron. Astrophys.'104, 203-206.

Bogod, V.M., Vyalshin, G.F., GeIfriekh, G.B., and Petrova, N.W.: 1982, Soln. Dann. 1, 104.

Bonet, J.A., Ponz, J.D., and Vazquez, M.: 1982, Solar Phys. 77, 69-75. 
Brants, J.J. and Zwaan, C.: 1982, Solar Phys. 80, 251-258

Bumba, V. and Suda, J.: 1983a, Bull. Astron. Inst. Czech. 34, 349.

Bumba, V. and Suda, J.: 1983b, Bull. Astron. Inst. Czech. 34, 155.

Bumba, V. and Suda, J.: 1983c, Bull. Astron. Inst. Czech. 34, 29

Bumba, V. and suda, J.: 1984a, Bull. Astron. Inst. Czech. 35, 28.

Bumba, V. and Suda, J.: 1984b, Bull. Astron. Inst. Czech. 35, 224.

Bumba, V. et al.: 1981, Bull. Astron. Inst. Czech. 32, 286.

Bumba, V. et al.: 1982, Bull. Astron. Inst. Czech. 33, 160.

Cally, P.S.: 1983, Solar Phys. 88, 77-102.

Cally, P.S. and Adam, J.A.: 1983, Solar Phys. 85, 97-111.

Cram, L.E. and Thomas, J.H. (eds.): 1981, The Physics of Sunspots, Sacramento Peak Observatory, Sunspot, New Mexico.

Cram, L.E., Nye, A.H., and Thomas, J.H.: 1981, in Phys. of Sunspots, pp. $384-388$. deloach, A.C., Hagyard, M.J., Rabin, D., Moore, R.L., Smith, J.B., Jr., West, E.A., and Tandberg-Hanssen, E.: 1984, Solar Phys. 91, 235-242.

Denisenko, V.V., Kotov, V.A., Romanov, V.A., and Sokolov, V.S.: 1982, Solar Phys. 81, 217-229.

Dezsö, L. and Kalman, B. (eds.): 1983, Proc. 11th Regional Consultation on Solar Physics, Publ. Debrecen Obs. 5.

Dezsö, L. and Kovacs, A.: 1983, Publ. Debrecen Heliophys. Obs. 5, 317-322.

Doval, P.: 1983, Soln. Dann. 12, 81.

Elboth, D. and Engvold, O.: 1983, Rep. Inst. Theoret. Astrophys., Blindern, Oslo $59,15$.

Flaa, T., Osherovich, V.A., and Skumanich, A.: 1982, Astrophys. J. 261, 700.

Fowler, L.A., Foukal, P., and Duvall, T., Jx.: 1983, Solar Phys. 84, 33-44.

Garcia de la Rosa, J.I.: 1981, Solar Phys. 74, 117-123.

Garcia de la Rosa, J.I.: 1983, Solar Phys. 89, 51-62.

Giovanelli, R.G. and Jones, H.P.: 1982, Solar Phys. 79, 267-278.

Glenar, D.A., Deming, D., Jennings, D.E., Kostiuk, T., and Mumma, M.J.: 1983, Astrophys. J. 269, 309-318.

Godoli, G. and Mazzucconi, F.: 1982, Astron. Astrophys. 116, 188-189.

Gopasyuk, S.I.: 1981, Izv. Krymsk. Astrofiz. Obs. 64, 108.

Gopasyuk, S.I.: 1982, Izv. Krymsk. Astrofiz. Obs. 66, 77.

Gopasyuk, S.I. and Kartashova: 1981, Izv. Krymsk. Astrofiz. Obs. 63, 25.

Gurman, J.B.: 1984, Solar Phys. 90, 13-15.

Gurman, J.B., Ieibacher, J.W., Shine, R.A., Woodgate, B.E., and Henze, W.: 1982, Astrophys. J. 253, 939-948.

Hagyard, M.J., Teuber, D., West, E.A., Tandberg-Hanssen, E., Henze, W., Jr., Beckers, J.M., Bruner, M., Hyder, C.L., and Woodgate, B.E.: 1983, Solar Phys. 84, 13-31.

Hauge, O. (ed.): 1983, Rep. Inst. Theoret. Astrophys., Blindern, Oslo 59.

Henze, W., Jr., Tandberg-Fanssen, E., Hagyard, M.J., Woodgate, B.E., Shine, R.A., Beckers, J.M., Bruner, M., Gurman, J.B., Hyder, C.L., and West, E.A.: 1982, Solar Phys. 81, 231-244.

Henze, W., Tandberg-Hanssen, E., Reichmann, E.J., and Athay, R.G.: 1984, Solar Phys. 91, 33-44.

Howard, R., Gilman, P.A., and Filman, P.I.: 1984, Astrophys. J. 283, 373-384.

Hudson, H.S., Silva, S., and Woodward, M.: 1982, Solar Phys. 76, 211-219.

Huseynov: 1982, Izv. Krymsk. Astrofiz. Obs. 66, 83.

Huseynov: 1983, Izv. Krymsk. Astrofiz. Obs. 68, 36.

Huseynov and Russkih: 1983, Izv. Krymsk. Astrofiz. Obs. 68, 31.

Ikhsanov, V.N.: 1982, Soln. Dann. 11, 88.

Karlicky, M. and Suda, J.: 1983, Bull. Astron. Inst. Czech. 34, 282-285.

Kawakami, H.: 1983, Publ. Astron. Soc. Japan 35, 459-489.

Kemp, J.C. and Henson, G.D.: 1983, Astrophys. J. Letters 266, L69.

Kingston, A.E., Doyle, J.G., Dufton, P.L., and Gurman, J.B.: 1982, Solar Phys. $81,47-58$.

Koch, A.: 1984, Solar Phys. 93, 53-72.

Koval, I.K. and stepanjan, N.N.: 1983, Izv. Krymsk. Astrofiz. Obs. $68,3$. 
Kovitya, P. and Cram, I.: 1983, Solar Phys. 84, 45-48.

Krivodubskij: 1983, Soln. Dann. 11, 51.

Kǔ̌era: 1982, Bull. Astron. Inst. Czech. 33, 345.

Lang, K.R. and Willson, R.F.: 1982, Astrophys. J. Letters 255, L111-L117.

Lawrence, J.K.: 1983, Solar Phys. 87, 1-5.

Lee, S. - W., et al.: 1982a, J. Korean Astron. Soc. 14, 19.

Lee, S. - W., et al.: 1982b, J. Korean Astron. Soc. 14, 79.

Iiggett, M. and Zirin, H.: 1983, Solar Phys. 84, 3-11.

Lin, R.P. and Wang, J.-1.: 1982, Sci. Sinica Series A 25, 89.

Lites, B.W.: 1984, Solar Phys. 90, 1-12.

Lites, B.W. and Skumanich, A.: 1981, in Phys. of Sunspots, pp. 152-166.

Lites, B.W., White, O.R., and Packman, D.: 1982, Astrophys. J. 253, 386-392.

Loskutnikov: 1984, Soln. Dann. 6, 91.

Moore, R.L.: 1981a, Space Sci. Rev. 28, 387-421.

Moore, R.I.: 1981b, Astrophys. J. 249, 390-393.

Mooxe, R.L.: 1981c, in Phys. of Sunspots, pp. 259-311.

Mordvinov: 1983, Soln. Dann. 5, 76.

Muller, R.: 1981, in Phys. of Sunspots, pp. 340-342.

Nicolas, K. R.: 1981, in Phys. of Sunspots, pp. 336-339.

Nicolas, K. R., Kjeldseth-Moe, O., Bartoe, J.-D., and Brueckner, G.E.: 1982, Solar Phys. 81, 253-280.

Obashev, S.0., Gainullina, R.K., Minasyants, T.M., and Minasyants, G. S.: 1982, Solar Phys. 78, 59-66.

Osherovich, V.A.: 1982, Solar Phys. 77, 63-68.

Osherovich, V.: 1984, Solar Phys. 90, 31-35.

Osherovich, V. and Flaa, T.: 1983, Solar Phys. 88, 109-115.

Osherovich, V.A. and Lawrence, J.K.: 1983, Solar Phys. 88, 117-135.

Parfinenko, L.D.: 1981a, Soln. Dann. 2, 96.

Parfinenko, I.D.: 1981b, Soln. Dann. 12, 79.

Parfinenko, L. D.: 1982, Soln. Dann. 5, 86.

Parfinenko, L.D.: 1983, Soln. Dann. 3, 102.

Patty, S. R.: 1981, in Phys. of Sunspots, pp. 64-74.

Schatten, K.H: 1981, Astrophys. J. Letters 247, L139-L142.

Selezneva, L.M.: 1981, Soln. Dann. 9, 94.

Shibasaki, K., Chiuderi-Drago, F., Melozzi, M., Slottje, C., and Antonucci, E.: 1983, Solar Phys. 89, 307-321.

Singh, P.D. et al: 1983, Acta Astron. Sinica 89, 207.

Sinha, R.P.: 1983, Bull. Astron. Soc. India 10, 223.

Sinha, R.P. and Joshi, M.N.; 1983, Bull. Astron. Soc. India 10, 329.

Solovev, A.A.: 1981, Soln. Dann. 12, 71.

Solovev, A.A.: 1982a, Astron. Zh. 59, 380 .

Solovev, A.A.: 1982b, Soln. Dann. 1, 86.

Solovev, A.A.: 1983, Soln. Dann. 3, 65.

Solovev, A. A.: 1984, Soln. Dann. 1, 73 .

Soltan, D.: 1981, Astron. Astrophys. 107, 211.

Spruit, H.C.: 1981a, in Phys. of Sunspots, pp. 359-368.

Spruit, H.C.: 1981b, Space Science Rev. 28, 435-448.

Stachnik, R.V., Nisenson, P., and Noyes, R.W.: 1983, Astrophys. J. Letters 271, L37.

Staude, J.: 1981, Astron. Astrophys. 100, 284-290.

Stellmacher, G. and Wiehr, E.: 1981, Astron. Astrophys. 103, 211-215.

Stenflo, J.O. (ed.): 1983, IAU Symp. No. 102, D. Reidel Publ. Co., Dordrecht.

Tang, F.: 1983, Solar Phys. 89, 43-50.

Teplitskaya, R.B. and Turova, I.P.: 1983, Soln. Dann. 8, 102.

Thomas, J.H.: 1981, in Phys. of Sunspots, pp. 345-358.

Thomas, J.H. and Scheuer, M.A.: 1982, Solar Phys. 79, 19-29.

Thomas, J.H., Cram, L.E., and Nye, A.H.: 1982, Nature 297, 485.

Thomas, J.H.: 1984, Astron. Astrophys. 135, 188.

Tönjes, K. and Wöhl, H.: 1982, Solar Phys. 75, 63-69. 
Tuominen, J. and Kyröläinen, J.: 1982, Solar Phys. 79, 161-172. Turova, I.P.: 1984, Solar Phys. 91, 51-54. Turova, I.P., Teplitskaja, R.B., and Kuklin, G.V.: 1983, Solar Phys. 87, $7-22$. Van Ballegooijen, A.A.: 1984, Solar Phys. 91, 195-217. Vyalshin, G.F. and Kulagin, E.S.: 1981, Soln. Dann. 9, 97. Wallenhorst, S.G. and Howard, R.: 1982, Solar Phys. 76, 203-209. Wallenhorst, S.G. and Topka, K.P.: 1982, Solar Phys. 81, 33-46. West, E.A. and Hagyard, M.J.: 1983, Solar Phys. 88, 51-64. Wöhl, H., Engvold, O., and Brault, J.W.: 1983, Rep. Inst. Theoret. Astrophys., Blindern, Oslo 56.

Wittmann, A.: 1983, Mitt. Astron. Ges. 60, 280.

Yang, H.-S., Chang, H.-M., and Harvey, J.W.: 1983, Solar Phys. 84, 139-151. Ye, S. - H. and Jin, W.-J.: 1981a, Acta Astron. Sinica 22, 365. Ye, S.-H. and Jin, W.-J.: 1981b, Acta Astron. Sinica 23, 276. Yoshimura, H.: 1983, Solar Phys. 87, 251-259.

Yun, H.S. and Beebe, H.A.: 1982, Solar Phys. 78, 347-349.

Yun, H.S. and Kim, Y.H.: 1983, J. Korean Astron. Soc. 16, 19.

Yun, H.S., Beebe, H.A., and Baggett, W.E.: 1982, Proc. Southwest Reg. Conf., Astron. Astrophys. 8, 31.

Yun, H.S., Beebe, H.A., and Baggett, W.E.: 1984, Solar Phys. 92, 145-151.

Zhugzhda, Y.D. and Makarov, V.I.: 1982, Solar Phys. 81, 245-251.

Zhugzhda, Y.D., LoCans, V., and Staude, J.: 1983, Solar Phys. 82, 369-378.

Zhugzhda, Y.D., Staude, J., and Locans, V.: 1984, Solar Phys. 91, 219-234.

VI. Solar Radiophysics

A. INTRODUCTION

The past 3 years since the last solar maximum have witnessed an unprecedented number $(>200)$ of published scientific papers on many aspects of solar radiophysics. These contributions are the result of an intense research effort mounted during the first Solar Maximum Mission of 1980 and continued until the present. Excellent x-ray, EUV, and visible light observations of the disturbed corona and transition region have been obtained from the SMM, Hinotori, P78-1, and ISEE-3 spacecraft. ISEE-3 also has provided very Iow-frequency radio observations of solar bursts in the interplanetary medium. Ground-based radio support for space experiments has been provided by many observatories throughout the world. In particular, many collaborative studies using $x-r a y$ and radio observations of solar flares have been reported. The outstanding radio instrument during this period has been the VLA, operating at 2, 6 , and $20 \mathrm{~cm}$ with a time resolution of $10 \mathrm{~s}$ and both modes of circular polarization. The twodimensional spatial resolution of the radio images is a few seconds of arc, almost as good as the best resolution obtained so far at any wavelength in the solar spectrum. To complement the rather poor time resolution of the VLA onedimensional arrays such as the WSRT at $6 \mathrm{~cm}$ wavelength and the Nobeyama interferometer at $17 \mathrm{GHz}$ have been used successfully. In addition, a number of very-high-time resolution radiometers have been built at different locations. At meter wavelengths two-dimensional arrays at Clark Lake, Culgoora, and Nancay and a one-dimensional array at Nobeyama have been employed. The little known region of the solar spectrum at decimeter wavelengths is being investigated by the zürich spectrograph. It is pleasing to see Chinese participation in solar radiophysics.

Looking ahead there remains only one part of the solar spectrum, from 20 to 1 MHz and corresponding to coronal heights from 3 to $30 \mathrm{R}_{0}$, to be explored. This spectral gap hinders the merging of coronal and interplanetary radiophysics. Another obvious need in the future is for a high time and spatial resolution radioheliograph operating at microwave frequencies, i.e., near $17 \mathrm{GHz}$. Such an instrument combined with high spatial hard $x$-ray observations could settle some 